\title{
An investigation of the porous silicon optical absorption power law near the band edge
}

\author{
P M Derlet $\dagger$, T C Choy $\dagger$ and A M Stoneham \\ $\dagger$ Department of Physics, Monash University, Clayton, Victoria 3168, Australia \\ $\ddagger$ AEA. Technology, Harwell Laboratory, Oxfordshire OX11 ORA, UK
}

Received 26 August 1994, in final form 5 December 1994

\begin{abstract}
A theoretical investigation of the absorption coefficient of p-type doped porous silicon near the band edge is presented. We assume that the absorption coefficient is constructed by taking an average over a distribution (in terms of band gap) of absorption coefficients of individual crystallites. Exploiting physics fundamental to the crystallite optical absorption process, we derive the relation between the absorption coefficient and the averaged conduction density of states near the band edge for porous silicon. By postulating a specific form for the effective conduction density of states we find excellent agreement with recent optical absorption data for p-type doped porous silicon. We attempt to explain the basis for this postulate phenomenologically by suggesting a certain large-scale behaviour of the particle size distribution. The implication of further experimental verification will be discussed.
\end{abstract}

\section{Introduction}

The precise structural topology of p-type porous silicon is difficult to determine and characterize. What is known is that (up to) $80 \%$ of the silicon atoms are removed by the anodization process and what remains is an interconnected (noodle-like) network of quantum crystallites and wires of undulating thickness [1-4]. The exact distribution of shapes and sizes is often sample dependent, relying on the doping level (and type) of the bulk silicon and on the anodization process. For n-type porous silicon the situation is somewhat simplified, the general structure consisting of quantum wires that are predominantly along the [100] crystallographic direction and which do not interconnect [5].

From a theoretical perspective, porous silicon is unique in that it is perhaps the first realistic quantum system (solid) that contains both disorder and order on separate length scales. The order lies in the regular nano-sized silicon structure that remains after anodization and the disorder originates in its shape and size distribution. Over the past two years there has been an extensive theoretical investigation on the electronic properties of quantum wires and crystallites; the average over differing crystallite sizes, reflecting the measurable properties of porous silicon.

In the present paper we concentrate on the optical absorption coefficient for p-type porous silicon. Sagnes et al [6] have undertaken a thorough investigation of the optical absorption coefficient for $\mathrm{p}$-type and $\mathrm{p}^{+}$-type porous silicon. Via optical transmission data, they provided detailed structure near the (blue-shifted) band edge for a number of different porosities and deduce a number of properties such as crystallite distribution and the effect of p-type doping. 
Unnoticed by them, a clear power law for the band-edge absorption emerges, quite different from that associated with the usual bulk indirect and direct absorption mechanisms. Within this conventional framework, the data of Sagnes suggest (albeit tenuously) that the absorption mechanism may be more closely related to the 'forbidden (phonon-assisted) indirect' process (corresponding to a cube law for the absorption coefficient with respect to incident photon energy). This, however, must be precluded due to its extremely low quantum efficiency. We are therefore motivated to investigate the effect that disorder has on the band-edge absorption of porous silicon since an uncommon power law suggests that the effective density of states for porous silicon differs from the usual square root form of simple three-dimensional systems.

Sagnes comment that the absorption curves can be interpreted either in terms of a bandgap distribution or in terms of a roughly single large band gap that is 'smeared' out by a 'large defect concentration in the gap'. We shall mainly consider the former in this paper.

In section 2 of this paper we first discuss the fundamental physics of the optical absorption process for a single crystallite. Using this, we derive the relation between the optical absorption coefficient of porous silicon and its averaged conduction density of states near the band edge. The latter we shall assume to be an average over the relevant (confinement) lengths of the crystallites that constitute porous silicon or equivalently, an average over a band-gap distribution; there being a one-to-one correspondence between the two. This definition is not unique however. If the picture of defect concentration in the gap is used, then we should define this as an average over defects. Nevertheless by postulating a quadratic law for the averaged density of states in section 3, we find excellent agreement with the optical absorption data of Sagnes et al. In section 4 we seek an explanation for the origin of the quadratic density of states postulate. The picture we explore is phenomenological in that, via a plausibility argument incorporating aspects of percolation theory, we infer a direct relationship between the averaged density of states and the confinement length (band-gap) distribution function. Such a picture obviously requires further experimental verification by studying particle size distribution using, for example, a scanning electron microscope. If correct, then this aspect of the band-edge behaviour of porous silicon is basically a result of the anodization (electrochemical) process. On the other hand, if incorrect, the quadratic power law in the density of states may indeed be fundamental to the physics of porous silicon and hence of deeper significance. These matters will be discussed in section 5 .

\section{An optical absorption model for porous silicon}

To obtain the absorption coefficient for a particular crystallite as a function of incident photon energy, sophisticated silicon cluster calculations are needed. We, however, are only interested in its form near the band edge. Fortunately this considerably simplifies the calculation by an effective-mass approach which as we shall see, still describes the essential physics of the absorption mechanism for both single crystallites and porous silicon up to nearly $1 \mathrm{eV}$ beyond the band edge.

Since porous silicon is structurally sound (i.e. it does not fall apart after anodization) there exist extended regions in many directions in addition to the local dominant crystallite. This entails a three-dimensional continuum of states with energies above that of the local (upshifted) band gap. The resulting local density of states near the new band edge will therefore arise from a quasi-three-dimensional system and will not be that different from the usual square root law of the three-dimensional continuum.

Further evidence is suggested by $[7,8]$, wherein it is inferred via a simple zone-folding argument that nano-sized silicon structures are approximately direct-gap semiconductors. 
Hence, for our purposes, the band structure near the band edge for the $j$ th crystallite (we again emphasize that, by this, we mean a region characterized by a particular confinement length) can be adequately constructed from an upside down quadratic valence band: $E_{\mathrm{v}}(k)=-\hbar^{2} k^{2} / 2 m_{\mathrm{v}} ;$ and a quadratic conduction band: $E_{\mathrm{c}}^{J}(k)=\hbar^{2} k^{2} / 2 m_{\mathrm{c}}+E_{\mathrm{g}}^{j}$ where $E_{\mathrm{g}}^{j}$ is the local band gap. The corresponding valence and conduction local density of states are, respectively, $\rho_{\mathrm{v}}(E)=\left(2 m_{\mathrm{v}} / \hbar^{2}\right)^{3 / 2} \sqrt{-E} / 2 \pi^{2}$ and $\rho_{\mathrm{c}}^{j}(E)=\left(2 m_{\mathrm{c}} / \hbar^{2}\right)^{3 / 2} \sqrt{E-E_{\mathrm{g}}^{j}} / 2 \pi^{2}$. Thus the valence density of states is the same for all crystallites. Regardless of the model, this can be generally justified by assuming that the core valence electron wavefunctions in porous silicon are not significantly altered from that of the bulk-an underlying assumption of the recent tight-binding calculation for crystallites and wires.

For very small (smaller than average) crystallites (typically $2-4 \mathrm{~nm}$ ) the assumption of a continuum of states may be invalid or weakened. Indeed a discrete energy spectrum is expected. Such crystallites would not contribute significantly to the band-edge absorption because it is the larger than average crystallites (typically $6-10 \mathrm{~nm}$ ) that dominate the band edge. In addition, experiment does not seem to show any discrete structure-although the instrumental function of the apparatus may not resolve such structure.

In addition to the direct-gap nature of the crystallites, the lack of translational invariance removes the requirement for momentum conservation, giving an absorption mechanism quite different from that of the bulk silicon case. It can be shown (see the appendix) that for porous silicon, the optical (band-edge) absorption coefficient is approximated well by

$$
\alpha_{\mathrm{PS}}(\hbar \omega) \sim \frac{1}{\hbar \omega} \int \rho_{\mathrm{v}}(E) \rho_{\mathrm{c}}^{\mathrm{PS}}(E+\hbar \omega) \mathrm{d} E
$$

where

$$
\rho_{\mathrm{c}}^{\mathrm{PS}}(E)=(1 / N) \sum_{j} \rho_{\mathrm{c}}^{j}(E)
$$

is an average of the density of states over $N$ crystallites. Equations (1) and (2) imply that the non-local electron density of states that the optical photons 'see' is an effective density of states which need no longer follow a square root power law.

The physics of this absorption process is fundamental and generalizes from the optical properties of bulk semiconductors [9]. What follows is our postulate that $\rho_{\mathrm{c}}^{\mathrm{PS}}(E)$ has the quadratic form $\rho_{\mathrm{c}}^{\mathrm{PS}}(E) \sim\left(E-E_{\mathrm{g}}^{\mathrm{min}}\right)^{2}$, where $E_{\mathrm{g}}^{\min }$ is the band gap of porous silicon. In the next section we shall show that it gives nearly perfect agreement with the absorption data of Sagnes et al which follows a power law of exponent $7 / 2$ in the absorption coefficient, unnoticed by these authors.

\section{Comparison with optical absorption data}

We now have an approximate relationship between the band-edge absorption curve and the conduction electron density of states. Through the experimental data of Sagnes, we have available to us an empirical base from which to determine the power law for the optical absorption. However, it is more instructive to consider a possible form for the effective density of states for the conduction electrons.

It is a general feature of the electronic density of states that for disordered or amorphous systems, tails occur at the band edge. This may either arise from topological or interstitial (doping) disorder. It is therefore not too bold to expect a similar band-tail phenomenon for porous silicon. For simplicity (justified in section 4), we assume the band-edge conduction density of states is described by a power law with an exponent greater than one. 
Without detailed knowledge gained through experiment it is difficult to go further in the determination of the exponent. However, if we begin with the postulate that the average density of states follows a quadratic power law at the band edge, we then obtain (using equation (1)) a 7/2-power law for the band-edge absorption:

$\alpha_{\mathrm{PS}}(\hbar \omega) \sim \frac{1}{\hbar \omega} \int_{E_{\mathrm{g}}-\hbar \omega}^{0} \sqrt{-E}\left(E_{\mathrm{g}}^{\min }-E-\hbar \omega\right)^{2} \mathrm{~d} E \sim \frac{1}{\hbar \omega}\left(\hbar \omega-E_{\mathrm{g}}^{\min }\right)^{7 / 2}$.

We have found that the 7/2-power law generally provided the best fit to the raw data at hand. Of course, the other avenue is to keep the exponent free and determine it from the best fit (as we have indeed done for a number of samples). By removing certain anomalies (to be discussed at the end of this section) we found that the fitted value of the exponent differed by no more than 10 to $20 \%$. However, often the resulting band gap was less than that of bulk silicon! We believe it is more productive to begin analyses from the former point of view and assume a quadratic density of states since it provides a starting point for our discussion and understanding.

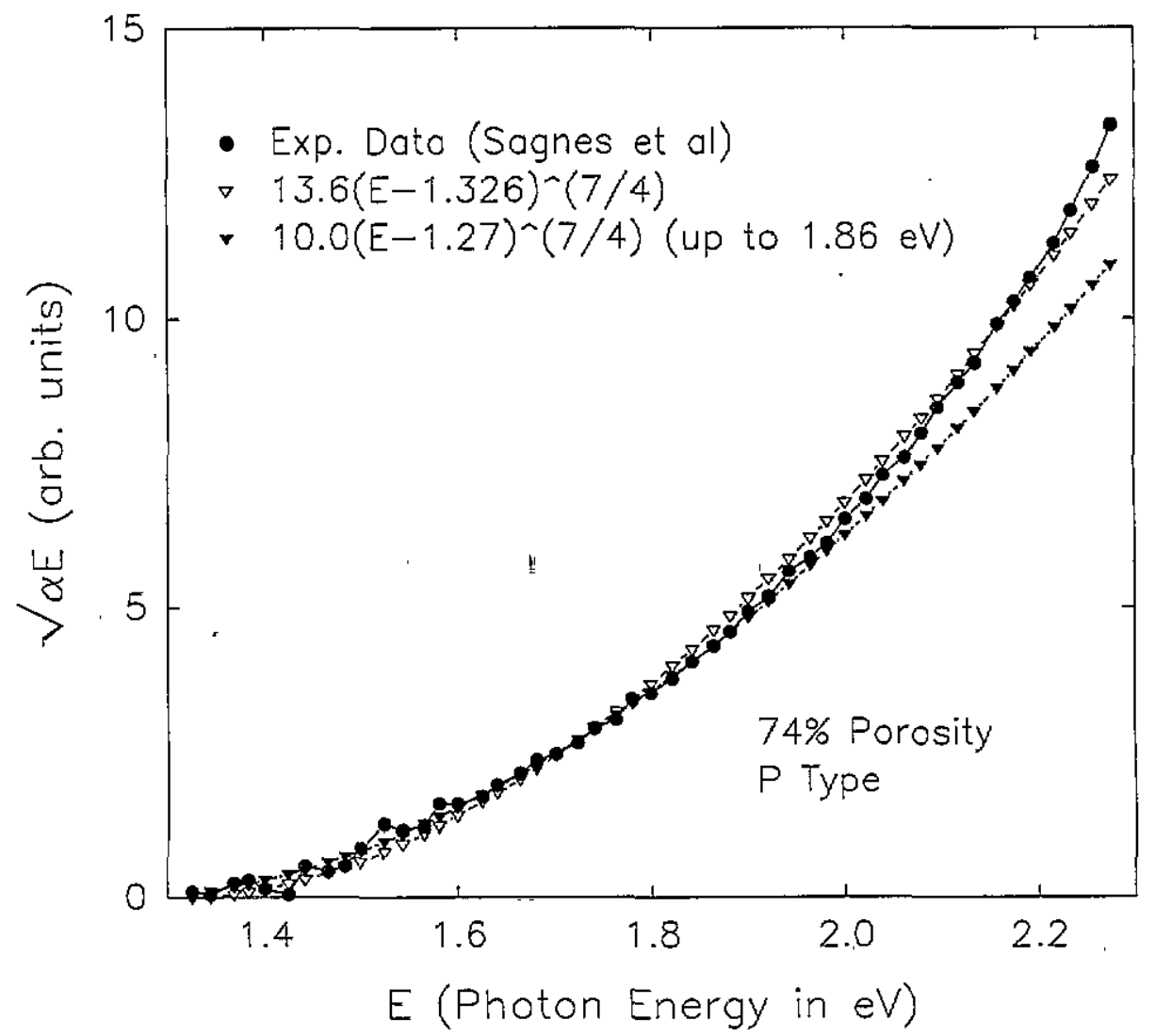

Figure 1. The optical absorption data measured by Sagnes et al [6] for p-type porous silicon with a porosity of $74 \%$ together with a fit to the $7 / 2$-power law. The noise in the experimental data below $1.6 \mathrm{eV}$ made it difficult to obtain a unique fit (in terms of band gap) which could be maintained for different sample regions of the data.

The absorption data of Sagnes clearly indicate a power law which at first glance extends almost one electron volt above the band gap of their porous silicon sample. Figures 1 (a 
porosity of $74 \%$ ) and 2 (a porosity of $58 \%$ ) are reproductions of their absorption data ( $\sqrt{h \omega \alpha}$ versus $\hbar \omega$ ) for p-type porous silicon obtained from transmission data and corrected for porosity. That is, the experimental absorption coefficient for porous silicon, $\alpha_{P S}$, is determined from the equation $T=T_{0} \exp \left(-\alpha_{\mathrm{PS}} d(1-P)\right.$ ) where $T_{0}(T)$ is the incident (transmitted) photon intensity, $d$ the thickness of the porous silicon sample, and $P$ its porosity. Also included in these figures is a numerical least-squares fit of the data to the 7/2-power law (the fit is in fact to a 7/4-power law due to the vertical axis being $\sqrt{\hbar \omega \alpha}$ ) where the initial free parameters are the band gap and the proportionality constant. For both figures the fit is performed using firstly all the absorption data and secondly only the data for photon energies of up to $1.8 \mathrm{eV}$. The latter is perhaps more relevant because we expect a (single) power law to hold only near the band edge.

However, inspection of figures 1 and 2 reveals that the 7/2-power law fits remarkably well for the complete range. This may be due to a feature observed in the appendix (using the results of [10])-that the absorption coefficient for an individual crystallite $\left(\sim(\hbar \omega)^{-1}\left(\hbar \omega-E_{\mathrm{g}}\right)^{2}\right)$ seems to be valid for photon energies up to $2.4 \mathrm{eV}$; i.e. up to $0.8 \mathrm{eV}$ past the onset of absorption. We defer further discussion of this to section 5 .

The absorption data for $\mathrm{p}^{+}$(heavily doped) porous silicon are shown in figures 3 and 4. The power law and band gap for this type of porous silicon are not that different from those for bulk silicon, which follows a linear absorption edge due to the dominant 'allowed indirect' absorption mechanism. This is expected as electron microscopy measurements made by Sagnes indicate that for $\mathrm{p}^{+}$porous silicon, the typical crystallite size is up to $20 \mathrm{~nm}$ whereas for p-type porous silicon, they found that the typical size was mainly less than $4 \mathrm{~nm}$.

For $\mathrm{p}^{+}$porous silicon with a porosity of $79 \%$ an adequate fit of the absorption data to the 7/2-power law could not be found (figure 3). On the other hand leaving the exponent as a free parameter for the least-squares fitting procedure gave a 1.185 -power law with an associated band gap of $1.209 \mathrm{eV}$. For the $\mathrm{p}^{+}$sample with a porosity of $45 \%$, a reasonable fit to the 7/2-power law was found, however with a band gap of $0.9935 \mathrm{eV}$-less than that of the bulk. A better fit was obtained by adjusting the exponent to 1.522 giving a corresponding band gap which was now slightly larger than $1 \mathrm{eV}$.

Given that the typical confinement length is so large, the applicability of our model to $\mathrm{p}^{+}$porous silicon must be questioned. Specifically, the strict application of the zone-folding argument may be inappropriate for such confinement lengths. Instead $\mathrm{p}^{+}$-type porous silicon may be more like an indirect-band-gap semiconductor giving a square power law near the band edge for the absorption coefficient which corresponds to a linear power law for the method of display in figures $i$ to 5 . For $\mathrm{p}^{+}$porous silicon with a porosity of $78 \%$ this was found, the absorption curve being almost linear (figure 3).

Hybertsen [11] and Xie et al [12] have undertaken an extensive theoretical and experimental investigation of the absorption of porous silicon films. For their experimental absorption data they obtain a good exponential fit to $\alpha_{\mathrm{PS}}$ versus $\hbar \omega$ beginning at a photon energy of approximately $1.6 \mathrm{eV}$ and including energies up to $2.8 \mathrm{eV}$. At first sight, comparison firstly with Sagnes absorption data and then our model remains difficult due to an exponential fit not having a definable (physical) band gap. If we replot their data as $\sqrt{\hbar \omega \alpha}$ versus $\hbar \omega$ (figure 5), inspection of their absorption data reveals that a 7/2-power law fits reasonably well and therefore their data actually are in agreement with our model.

An additional trend that has been observed in the absorption data of both [6] and [12] for the highly porous samples is a small anomaly at the band edge. This small 'bump' can be seen in figure 3 ( $\mathrm{p}^{+}$-type with porosity $78 \%$ ), to a lesser extent in figure 2 (p-type with porosity $58 \%$ ), and also in figure 5 (p-type with porosity $73 \%$ ). If we ignore instrumental 


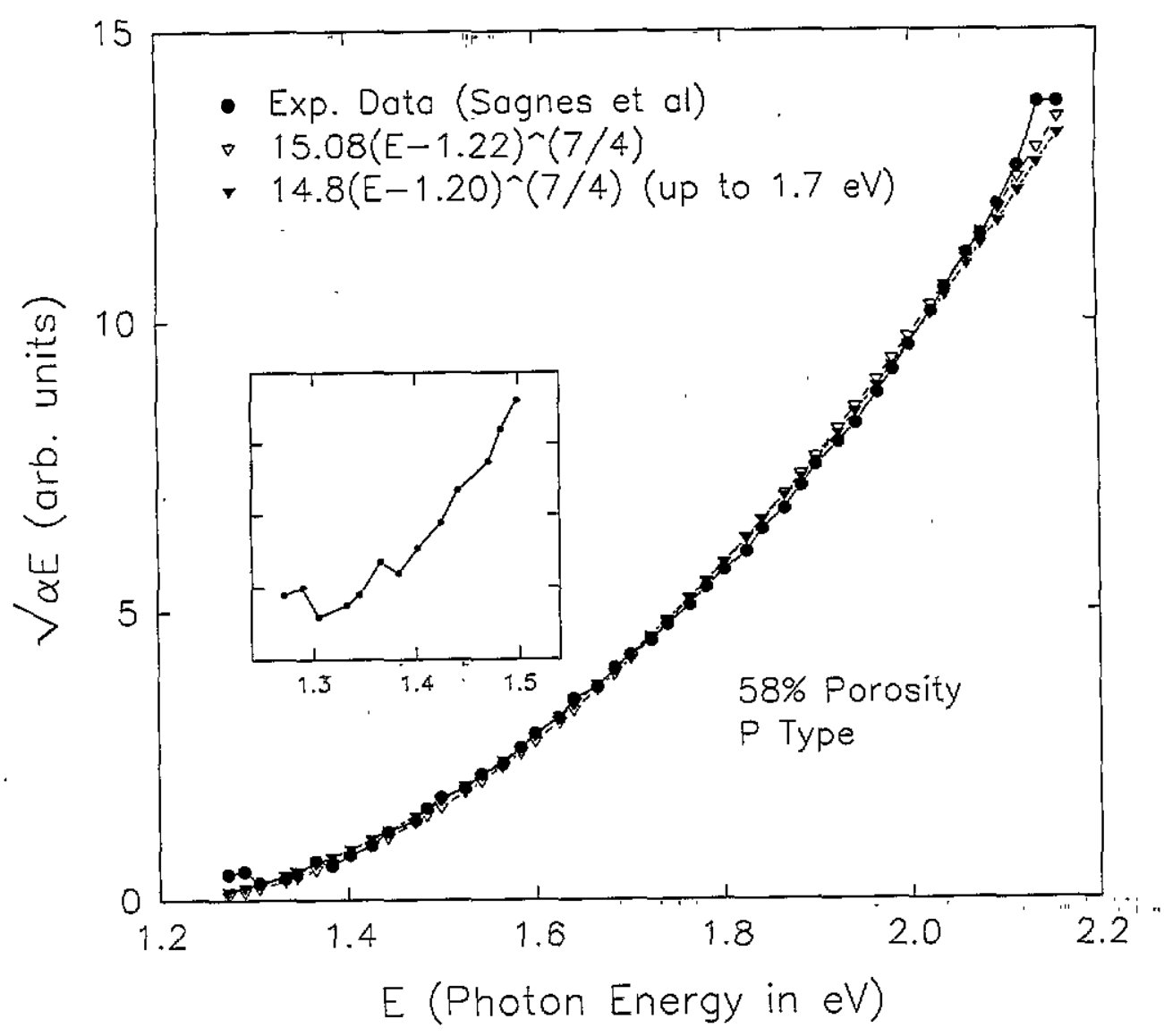

Figure 2. The optical absorption data measured by Sagnes et al [6] for p-type porous silicon with a porosity of $58 \%$ together with a fit to the 7/2-power law. When the fitting was performed without the first two data points (see inset) a 7/2-power law was easily fitted giving roughly the same fitting parameters (band gaps) for different sample regions of the data. The better fit (than figure 1) may be due to porosities in this range having larger (-than-average) crystallite sizes that are more appropriately stited to the approximations contained in our model.

error (we currently do not have such knowledge) this may be the signature of either a defect or surface state(s) of porous silicon. If it were surface states they would arise due to the macroscopic surface of porous silicon rather than say the microscopic surfaces of individual crystallites, the latter being smeared out due to the band-gap distribution.

\section{Confinement length and band-gap distribution functions}

Our present aim is to determine the properties of the crystallite distribution function that would account for the observed optical absorption data according to our viewpoint of a band-gap distribution, and thus to explain phenomenologically the origin of the quadratic density-of-states postulate.

If we consider any small region in porous silicon of dimensions in the tens of nanometres range, there will exist an average minimum length $R$ which characterizes the local dominant crystalline structure. This may be the average cross-sectional length of a quantum wire or the 


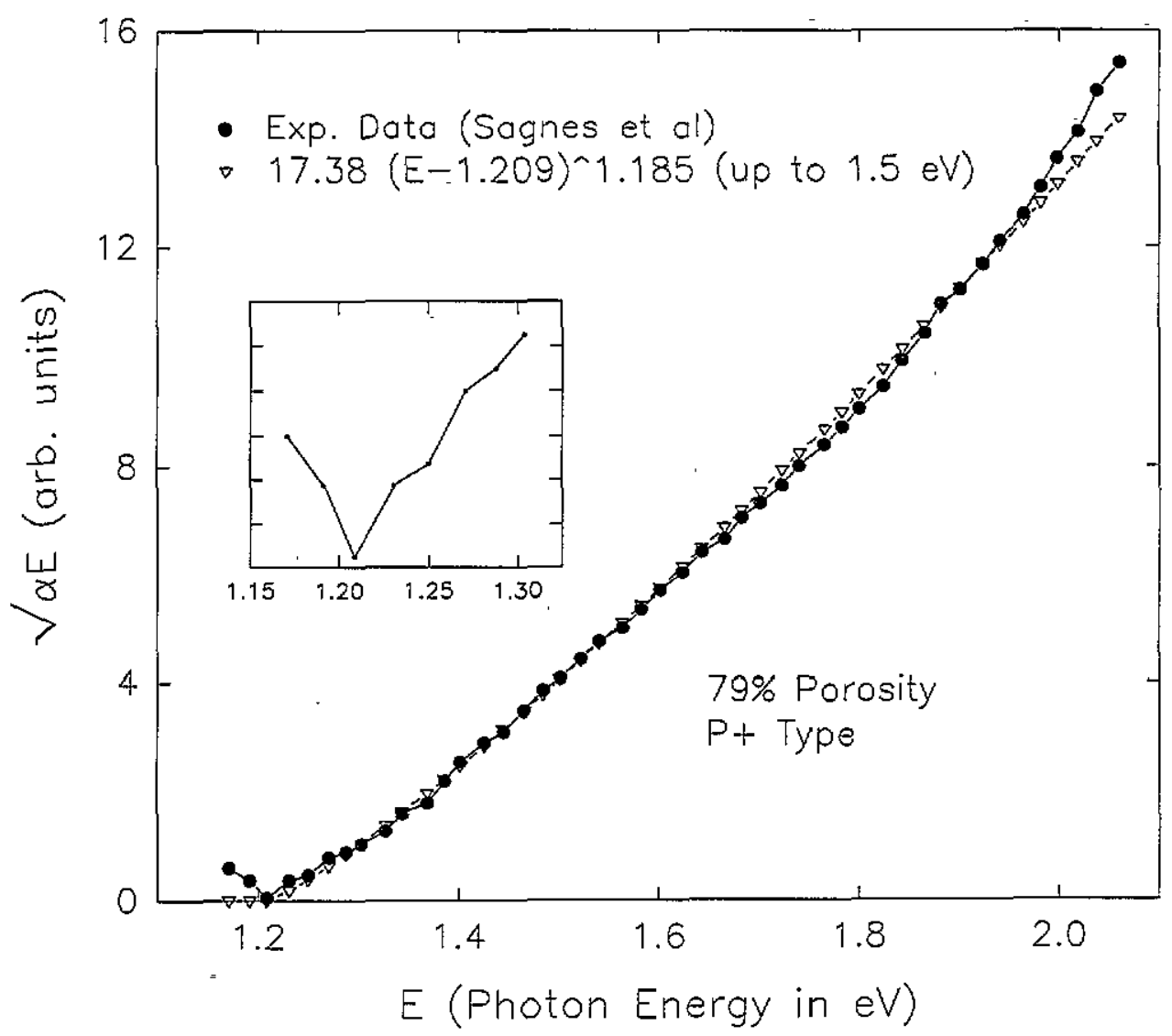

Figure 3. The optical absorption data measured by Sagnes et al [6] for $\mathrm{p}^{+}$-type porous silicon with a porosity of $79 \%$. A fit to the $7 / 2$-power law could not be obtained at all. The data did, however, fit well with an exponent of 1.185 , indicating that for this sample the absorption curve was more linear like that of an indirect-gap semiconductor. Again the small anomaly (see inset) at the band edge was not included in the data fit.

diameter of a dominant roughly spherical crystallite. Such small lengths lead to a quantum confinement effect where the zero-point energy of the conduction electrons is shifted up from the bulk silicon conduction band minima resulting in a larger band gap. From simple effective-mass theory this energy shift will go as $1 / R^{2}$. If the silicon band structure is taken into account (via the tight binding or linear combination of atomic orbitals framework; [13], [7] and [10]) the energy shift is found to obey the more general power law $1 / R^{\alpha}$ for radii in the nanometre range. Here $\alpha$ can range between 1.2 and $1.8[13,10]$ depending on the precise geometry of the structure. Through out the present work we use the term crystallite to represent the local dominant crystalline shape with confinement length $R$; i.e. we do not assume a specific crystalline shape for the microstructure of porous silicon.

We begin with $F(R)$ which represents the ratio between the number of crystallites per unit volume per unit confinement length and the total number of crystallites per unit volume, deriving the associated band-gap distribution using the relationship between confinement length and band gap. Since we 'wish to investigate the band-edge region of the optical absorption spectra, only the distribution of crystallites with smaller than average band gaps 


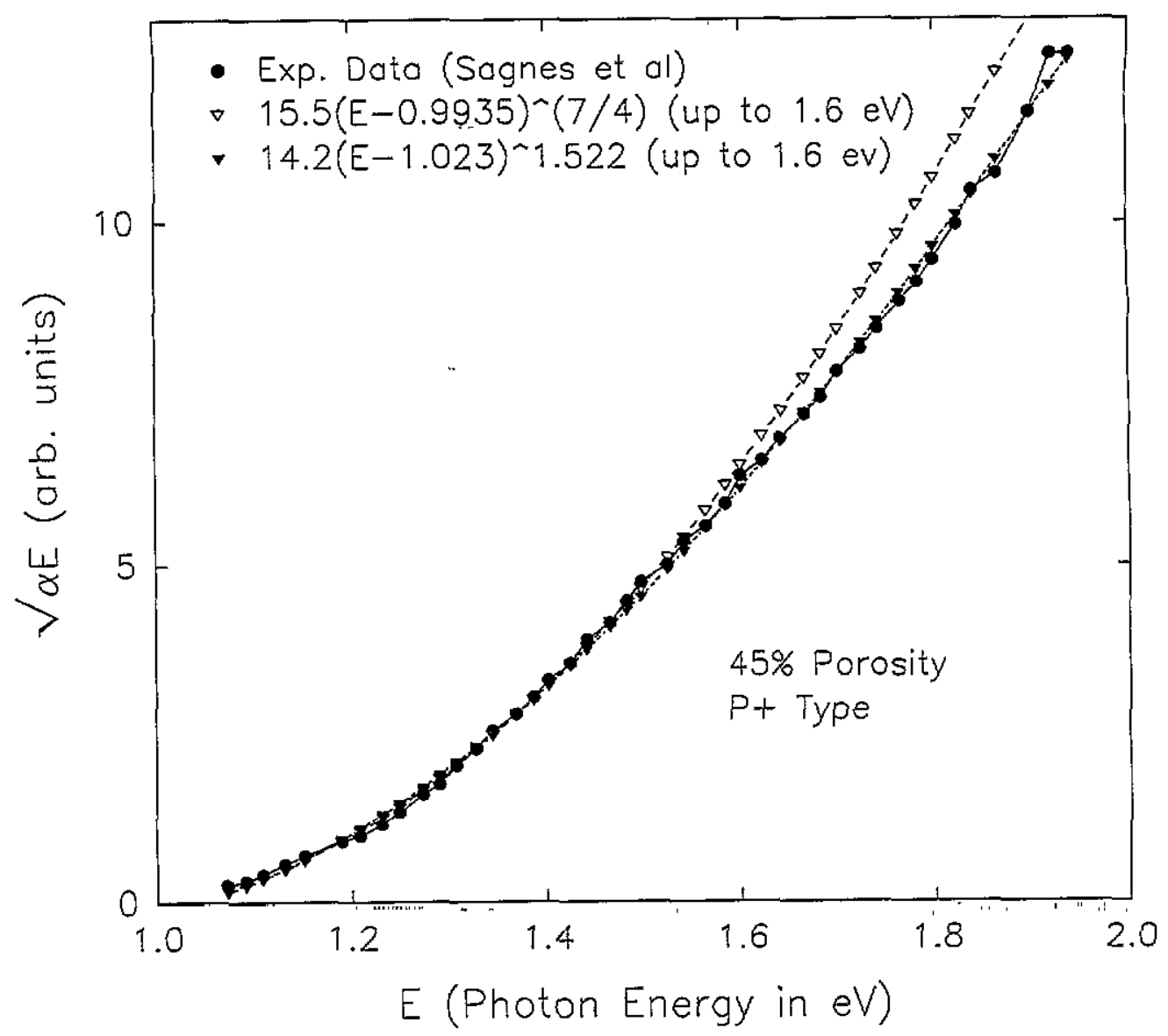

Figure 4. The optical absorption data measured by Sagnes et al [6] for $\mathrm{p}^{+}$-type porous silicon with a porosity of $45 \%$ together with a fit to the $7 / 2$-power law and also a fit where the exponent is left as a free parameter.

is needed. This corresponds to a knowledge of $F(R)$ for large $R$-considerably simplifying the problem at hand.

To gain an insight into $F(R)$ we begin with the very simplistic assumption that the anodization process can be viewed as a percolation problem-the action of etching represents the random removal of silicon atoms, where the probability that an atom is removed from a given site is defined to equal $p$. For an infinitely large system there exists a critical probability $p_{c}$ below which the voids (due to the removal of atoms) form finite-sized clusters and above which there exists at least one void that extends throughout the system. The function $F(R)$ will be associated with the remaining silicon structure.

For our purposes it is advantageous to begin our plausibility argument for $F(R)$ from a different (yet at this level of approximation, equivalent) percolation scenario. Here we start with an empty silicon lattice and randomly add atoms to it. In this case $p_{\mathrm{c}}$ would correspond to the critical state where there exists at least one silicon cluster of infinite size, extending throughout the system. Such a (single) cluster constitutes the majority of the porous silicon and the characteristic confinement lengths would arise from the distances between voids within this cluster. The morphology of such a cluster is in general a (finite) 


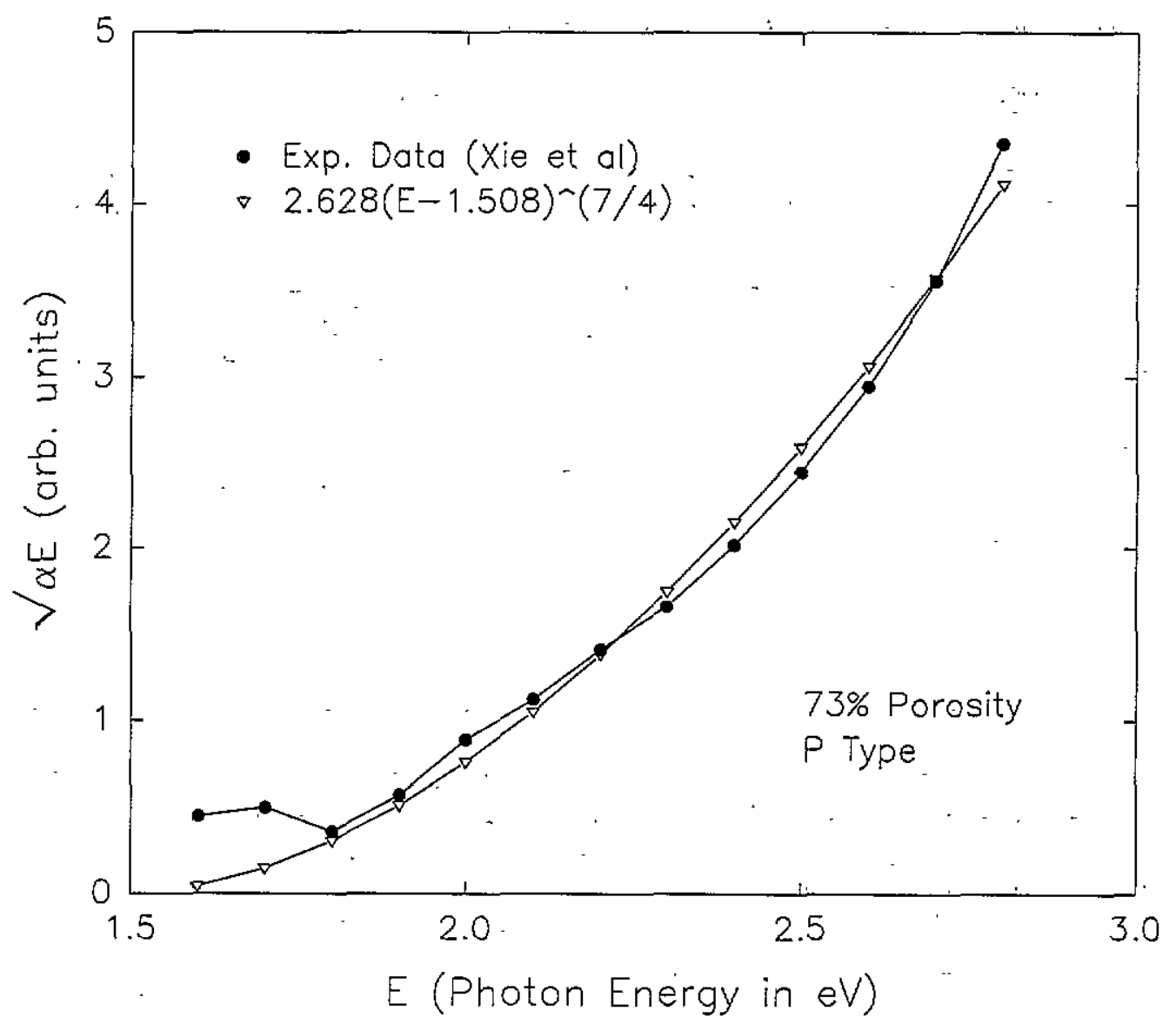

Figure 5. The optical absorption data measured by Xie et al [12] for p-type porous silicon with a porosity of $79 \%$ together with a fit (ignoring the anomaly) to the $7 / 2$-power law.

fractal and therefore approximately self-similar-it is well known that at least in terms of surface, porous structures are fractal like [14]. It is from this perspective that we begin with the proposition that asymptotically, $F(R)$ follows a simple power law behaviour; $F(R) \sim 1 / R^{\beta}$, where $\beta$ is an exponent yet to be determined. This is not an unusual assumption since a power law generally arises from self-similar structures.

In reality the actual formation of porous silicon is far more complex and less random than a simple percolation process, the etching beginning at the surface of the silicon substrate and being guided (it has been proposed) by the chemical passivation of the newly created surfaces [15] and/or the inhomogeneous resistivity/electric field of the remaining crystalline structure $[1,23]$ - both of which would control the direction of the local anodization current. This is the realm of the interacting percolation problem and hence it becomes very difficult to analyse qualitatively. For example there may no longer be a simple relationship between a percolation threshold and the porosity of the system. Nevertheless, for simplicity we still assume $F(R) \sim 1 / R^{\beta}$ for large $R$, although now this may be regarded as an approximate power law.

In addition, scanning electron microscope analyses of porous silicon reveal a crystallite size distribution which appears to be confined to the nanometre range $[2,6,17,18]$ indicating 
a (sample-dependent) maximum crystallite size. This is to be expected due to the general uniformity above the nanometre scale of the inhomogeneities of the pre-anodized bulk silicon surface. Such inhomogeneities are the catalysts for the chemical dissolution process $[1,23]$ and in part determine the maximum possible crystallite size-given that the growing pores (at least in p-type porous silicon) are generally interconnected. Such a maximum confinement length $R_{\max }$ corresponds to a minimum band gap $E_{\mathrm{g}}^{\min }$ larger than that of the bulk silicon band gap $E_{\mathrm{g}}^{\text {bulk. }}$.

To incorporate this into $F(R)$ we modify the $1 / R^{\beta}$ power law (which we now re-label as $\left.F_{\infty}(R)\right)$ by the additional factor $F_{\mathrm{c}}\left(R, R_{\max }\right)$ which is zero for $R \geqslant R_{\max }$ and has the limiting property $F_{\mathrm{c}}\left(R, R_{\max } \rightarrow \infty\right) \rightarrow 1$ for $R<R_{\max }$. The most general continuous function that has these properties is

$$
F_{\mathrm{c}}\left(R, R_{\max }\right)=\left(1-\left(\frac{R}{R_{\max }}\right)^{\gamma}\right)^{\nu} \quad(\nu>0)
$$

for $R<R_{\max }$, and

$$
F_{\mathrm{c}}\left(R, R_{\max }\right)=0
$$

for $R \geqslant R_{\max }$. Thus we have $F(R) \sim F_{\infty}(R) F_{\mathrm{c}}\left(R, R_{\max }\right)$, where

$$
F(R) \sim \frac{1}{R^{\beta}}\left(1-\left(\frac{R}{R_{\max }}\right)^{\gamma}\right)^{\nu}=\frac{1}{R^{\beta-\nu \gamma}}\left(\frac{1}{R^{\gamma}}-\frac{1}{R_{\max }^{\gamma}}\right)^{\nu} .
$$

Such a function occurs widely in physics when a finite measurable quantity is defined only with in an interval of an otherwise unbounded parameter. This is the simplest possible choice containing within it the explicit property that porous silicon has a band gap greater than that of bulk silicon. In reality there may exist an asymptotic 'tail' in the confinement length distribution function entailing that the increased band gap in porous silicon is only an approximate statement valid above a minimum optical transmission threshold.

The distribution of confinement lengths, $F(R)$, defines a distribution of crystallite band gaps. Using $E_{\mathrm{g}}=E_{\mathrm{g}}^{\text {bulk }}+1 / R^{\alpha}$ where $E^{\text {bulk }}$ is the band gap of bulk silicon (the numerator of the second term is set to unity for convenience-generally it depends weakly on the geometrical properties of the quantum-sized object), we have $R=\left(E_{\mathrm{g}}-E_{\mathrm{g}}^{\text {bulk }}\right)^{-1 / \alpha}$ and $\mathrm{d} R=-\left(E_{\mathrm{g}}-E_{\mathrm{g}}^{\mathrm{bulk}}\right)^{(\alpha+1) / \alpha} \mathrm{d} E_{\mathrm{g}} / \alpha$. In the same way, we can relate $R_{\max }$ to $E_{\mathrm{g}}^{\min }$ using $E_{\mathrm{g}}^{\min }=E_{\mathrm{g}}^{\text {bulk }}+1 / R_{\max }^{\alpha}$. We obtain for $R<R_{\max }$ and $E_{\mathrm{g}}>E_{\mathrm{g}}^{\min }$

$$
\begin{aligned}
& F(R) \mathrm{d} R=\frac{-1}{\alpha}\left(E_{\mathrm{g}}-E_{\mathrm{g}}^{\text {bulk }}\right)^{-(\alpha+1) / \alpha}\left(E_{\mathrm{g}}-E_{\mathrm{g}}^{\text {bulk }}\right)^{(\beta-v \gamma) / \alpha} \\
& \times\left[\left(E_{\mathrm{g}}-E_{\mathrm{g}}^{\text {bulk }}\right)^{\gamma / \alpha}-\left(E_{\mathrm{g}}^{\text {min }}-E_{\mathrm{g}}^{\text {bulk }}\right)^{\gamma / \alpha}\right]^{\nu} \mathrm{d} E_{\mathrm{g}} \\
&= G\left(E_{\mathrm{g}}\right) \mathrm{d} E_{\mathrm{g}}
\end{aligned}
$$

where $G\left(E_{\mathrm{g}}\right)$ is the distribution function for band gaps used in the appendix. Since we assume a minimum band gap larger than that of the bulk, we choose $G\left(E_{\mathrm{g}}\right)$ to be zero for $E_{\mathrm{g}} \leqslant E_{\mathrm{g}}^{\min }$. In its present form $G\left(E_{\mathrm{g}}\right)$ depends on the bulk band gap, an undesirable feature since within our model the bulk silicon band gap should no longer be a parameter of porous silicon. By requiring that $G\left(E_{\mathrm{g}}\right)$ be independent of $E_{\mathrm{g}}^{\text {bulk immediately implies }}$

$$
\frac{\alpha+1}{\alpha}=\frac{\beta-\nu \gamma}{\alpha}
$$

and

$$
\frac{\gamma}{\alpha}=1
$$


giving

$$
F(R) \sim \frac{1}{R^{\alpha+1}}\left(\frac{1}{R^{\alpha}}-\frac{1}{R_{\max }^{\alpha}}\right)^{\nu^{\prime}}
$$

and

$$
G\left(E_{\mathrm{g}}\right) \sim\left(E_{\mathrm{g}}-E_{\mathrm{g}}^{\mathrm{min}}\right)^{v}
$$

for the confinement length and band-gap distribution functions.

Although the $\beta$-coefficient (and hence the power law for large confinement lengths) no longer appears explicitly in equations (10) and (11), it does play a role implicitly by allowing the parameter $v$ to be regarded as free via equation ( 8 ).

In section 2 (and in the appendix) we assumed that the local density of states could still be represented by a square root power law. This approximation can now be made more rigorous by exploiting the mapping of the diffusion equation on a depleted silicon lattice (such as that produced by the percolation process) to the tight-binding (for example) Schrödinger equation. Alexander and Orbach [19] have determined the average autocorrelation function $\left\langle P_{0}(t)\right\rangle$ (single-site Green's function) for large times for a géneral system with fractal dimensionality $d$ and anomalous diffusion coefficient $\delta$, where the latter is defined through the large-time mean squared distance power law $\left\langle r^{2}(t)\right\}^{d / 2} \propto t^{2 /(2+\delta)}$. They find that $\left\langle P_{0}(t)\right\rangle \propto[V(t)]^{-1} \propto\left\langle r^{2}(t)\right\}^{d / 2} \propto t^{-d /(2+\delta)}$. The density of states for the diffusion problem is then $N(E)=-\operatorname{Im}\left\langle\tilde{P}_{0}\left(-E+\mathrm{i} 0^{+}\right)\right\rangle$where $\tilde{P}_{0}$ is the Laplace transform of $P_{0}(t)$. The resulting density of states for the diffusion problem becomes $\sim E^{d /(2+\delta)-1}$ which maps directly onto the conduction-band-edge local density of states, giving $\sim\left(E-E_{\mathrm{g}}\right)^{d /(2+\delta)-1}$; where $E_{\mathrm{g}}$ is the local band gap.

Thus from a knowledge of the dimensionality of the porous silicon lattice and the anomalous diffusion properties, the local band-edge density of states of interconnected crystallite structures can be found. Experimental investigation on the fractal dimension of porous silicon are rare; however, it is expected that its dimensionality would be slightly less than three. For a random walk on porous silicon, the porous regions would hinder the particles walk and therefore slow it down indicating that $\delta$ may be larger than zero. However, because there exist regions of order, $\delta$ will not be that different from zero. It is thus very plausible that as a first approximation, the local conduction density of states follows the usual square root power law at the local band edge defined by the local band gap.

Using equations (11) and (A7) immediately imply that the conduction density of states follows the power law:

$$
\rho_{\mathrm{c}}^{\mathrm{PS}}(E) \sim \int_{E_{\mathrm{g}}^{\operatorname{mun}}}^{E} \sqrt{E-E_{\mathrm{g}}}\left(E_{\mathrm{g}}-E_{\mathrm{g}}^{\min }\right)^{v} \mathrm{~d} E \sim\left(E-E_{\mathrm{g}}^{\min }\right)^{3 / 2+v} .
$$

and that a power law (using equation (1)) also emerges for the absorption coefficient of porous silicon,

$$
\begin{aligned}
\alpha_{\mathrm{PS}}(\hbar \omega) \sim & \frac{1}{\hbar \omega} \int_{E_{\mathrm{g}}-\hbar \omega}^{0} \rho_{\mathrm{v}}(E) \rho_{\mathrm{c}}^{\mathrm{PS}}(E+\hbar \omega) \mathrm{d} E \\
& \sim \frac{1}{\hbar \omega} \int_{E_{\mathrm{g}}-\hbar \omega}^{0} \sqrt{-E}\left(E+\hbar \omega-E_{\mathrm{g}}\right)^{3 / 2+v} \mathrm{~d} E \sim \frac{1}{\hbar \omega}\left(\hbar \omega-E_{\mathrm{g}}^{\mathrm{min}}\right)^{3+v} .
\end{aligned}
$$

We now have a relation (via equation (12)) between the average density of states of porous silicon and the confinement length distribution function for large $R$ or equivalently the band-gap distribution function for small $E_{\mathrm{g}}$. Our assumption of a quadratic density of 
states entails that the exponent $v$ is to equal $1 / 2$ thus giving a square root power law for the (smaller than average) band-gap distribution function. The vindication of the present phenomenological model thus lies in the experimental verification of equation (10) with $v=1 / 2$, and a suitable choice of $\alpha$.

From the present perspective a number of comments can now be made regarding the data of Sagnes. The observation (for p-type porous silicon) that the absorption power law is approximately the same for different porosities reflects that the precise form of the crystallite distribution is not strongly related to the porosity. However, since the band gap increases as the porosity increases (the least-squares fitting procedure gives a band gap equal to $1.27-1.326 \mathrm{eV}$ for a porosity of $74 \%$ (figure 1 ) and a band gap equal to $1.20-1.22 \mathrm{eV}$ for a porosity of $58 \%$ (figure 2)), the confinement length distribution $(F(R)$ ) does shift as a whole towards smaller lengths. This is in agreement with the statement by [6] that for p-type porous silicon, "the increasing porosities are obtained by a successive "chemical peeling' of the less porous structure".

In the case of $\mathbf{p}^{+}$porous silicon (specifically figure 4), a 7/2-power law could not be obtained. A better fit was obtained by allowing the exponent to be free, giving a value which was less than 7/2's. Such a power law entails $v<1 / 2$ which would imply a broader distribution of band gaps and hence confinement lengths. This is in agreement with gas adsorption isotherm experiments [6] which demonstrated that for a given substrate doping the average pore size increases and the pore distribution broadens entailing far fewer crystallites per unit volume.

\section{Discussion and concluding remarks}

We have found that the experimental optical absorption data of Sagnes support an approximate 7/2-power law and this in turn supports our postulate that the band-edge conduction density of states is approximately quadratic--an assertion that cannot yet be rigorously justified. However, using a phenomenological model we have related the quadratic density of states to the larger-than-average crystallite distribution function-an experimentally obtainable quantity.

In the context of our model the 7/2-power law dependence of the band-edge optical absorption requires an approximate square root form at the onset of the band-gap distribution, i.e. at $E_{\mathrm{g}}^{\min }$. If we assume the band-gap shift goes as $1 / R^{\alpha}$ where we take $\alpha=1.4$ [10], then with $v=1 / 2$, we have (using equation (10))

$$
F(R) \sim \frac{1}{R^{2.4}}\left(\frac{1}{R^{1.4}}-\frac{1}{R_{\max }^{1.4}}\right)^{1 / 2} .
$$

Equation (14) is valid only for large $R$ as our simple model can provide no information on the distribution for small (defined to be near and below the average crystallite size) $R$. However, the success of the model up to nearly an electron volt beyond the band edge suggests that this form for $F(R)$ is maintained into the region where the mean confinement length is located, suggesting that, in general, the complete confinement length distribution (whatever it may be) is skewed towards larger confinement lengths, having the approximate form $1 / R^{3.1}$ in the large- $R$ regime.

Although in our model we cannot make any absolute conclusions about $F(R)$ for small $R$, a simple skewed Gaussian would fall off much too quickly with respect to the location of its mean, whereas a log-normal distribution would fall off too slowly (which is always slower than $1 / R$ ). Furthermore, to incorporate a maximum confinement length (or equivalently a minimum band gap larger than that of the bulk case) simply into such 
distributions presents difficulties that cannot be easily and justifiably resolved given the available information of the system.

Nevertheless, we again emphasize that our power law is at this stage approximate and we cannot completely discount any such distribution of radii and hence band gaps. One must be careful in dismissing a particular model for crystal dissolution when comparing the associated predictions. with an experimental form of $F(R)$; for example, fragmentation models [20] which in general incorporate some aspects of percolation theory often predict log-normal distributions for cluster sizes. However, in fragmentation theory as in percolation theory, cluster size generally is defined as the number of sites (atom, molecules etc) that belong to that cluster, whereas the distribution for confinement lengths is derived from the internal structure of a particular cluster that extends through out the system; i.e. it becomes dependent on the degree of ramification, degree of self-similarity, and generally the nature of what is often referred to as the 'swiss cheese' model (the cheese constituting the remaining silicon) [21].

The issue of percolation also raises the possibility that the assumption that the measurable optical absorption is due to the average of the crystallites absorption coefficient is no longer valid. There may indeed exist a critical percolation state in which the 'typical' crystallite size diverges and an appropriate scaling procedure may be needed to describe the observed absorption accurately (as in the case for the metal-insulator transition in thin porous gold films [22]). Such a critical state will be characterized by the porosity, doping level and type, and detected by anomalous absorption data. This again suggests that a more detailed analysis is warranted.

The approximate square root power law for the band-gap distribution and its applicability up to nearly an electron volt beyond the band edge suggests that the true distribution is much broader than previously thought. The implications of such a broad distribution on the photoluminescence spectrum needs to be investigated since there is a belief that the full width at half-maximum of the peak is too large to be attributed entirely to a distribution of crystallite sizes [16] (this issue has also been disputed [24]). A detailed investigation of the photoluminescence properties using the present confinement length distributions is currently being undertaken and will be published in a subsequent paper.

A more direct experimental investigation is needed of the larger-than-average crystalline structure in p-type porous silicon to substantiate our model. We propose a systematic study of the microstructure as a function of porosity, doping level and type using scanning electron microscopy pictures in conjunction with image analysis software to characterize the nano-sized crystalline structure more accurately. In parallel, detailed optical absorption experiments are needed to investigate more closely the dependence of the absorption power law on these factors, and, we hope, to correlate our predictions with the micro-structural investigations. Also a more accurate investigation of optical absorption close to the vicinity of the band edge is required to verify the precise structure and origin of the anomaly we have cautiously attributed to either a defect or surface state(s).

If such an investigation verifies our phenomenological model, then the characteristics of the optical absorption process of porous silicon are due to a specific morphology which arises from the anodization process. If, however, experiment reveals a confinement length distribution which is quite different and more varied than that suggested by our model, the quadratic conduction density of states may arise from a more fundamental physical phenomena specific to porous silicon in which the local density of states is no longer quasithree-dimensional-no longer a square root power law-an underlying assumption of our 
modelt.

In conclusion we have proposed a simple model for the optical absorption coefficient of p-type porous silicon which provides a direct link to the average conduction density of states of porous silicon. By postulating a quadratic form at the band edge, excellent agreement is found using the optical absorption data provided by [6]. A phenomenological model is then used to relate the quadratic density of states to a confinement length (bandgap) distribution function, the consequences of which, are in general agreement with the conclusions of Sagnes et al.

\section{Acknowledgment}

This work was supported by an Australian Research Council Fellowship.

\section{Appendix. Derivation of the optical absorption coefficient for porous silicon}

Without the dynamical aspects of quantum confinement, the zone-folding argument implies that the dominant absorption mechanism at the band edge would be 'allowed direct' as in GaAs. That is,

$$
\begin{aligned}
\alpha^{j}(\hbar \omega) \sim & \frac{1}{\hbar \omega} \int \mathrm{d}^{3} k_{\mathrm{i}} \int \mathrm{d}^{3} k_{\mathrm{f}}\left|M_{k_{i}, k_{\mathrm{f}}}^{j}\right|^{2} \delta^{3}\left(k_{\mathrm{i}}-k_{\mathrm{f}}\right) \delta\left(E_{\mathrm{v}}\left(k_{\mathrm{i}}\right)-E_{\mathrm{c}}^{j}\left(k_{\mathrm{f}}\right)-\hbar \omega\right) \\
& \sim \frac{\left|M^{j}\right|^{2}}{\hbar \omega} \sqrt{\hbar \omega-E_{\mathrm{g}}^{j}}
\end{aligned}
$$

where we have assumed that the matrix element is independent of the initial and final momentum near the band edge; i.e. $\left|M_{k_{i}, k_{c}}^{j}\right|^{2}=\left|M^{j}\right|^{2}$. However, with quantum confinement the wave-vector is no longer a good quantum number and therefore is not conserved. As a first approximation this can be dealt with by replacing the wave-vector-conserving delta function with a function appropriate to the local crystalline shape centred on $k_{\mathrm{i}}-k_{\mathrm{f}}$, namely $h\left(k_{i}-k_{f}\right)$. For example if we assume the local crystalline shape is rectangular and orientated along the wave-vector basis vectors, then $h\left(k_{\mathrm{i}}-k_{\mathrm{f}}\right)$ would be a three-dimensional 'Sinc' function;

$$
h\left(k_{\mathrm{i}}-k_{\mathrm{f}}\right)=\frac{\sin \left(L_{x}^{j}\left(k_{\mathrm{ix}}-k_{\mathrm{f} x}\right)\right)}{k_{\mathrm{ix}}-k_{\mathrm{f} x}} \frac{\sin \left(L_{y}^{j}\left(k_{\mathrm{i} y}-k_{\mathrm{fy}}\right)\right)}{k_{\mathrm{i} y}-k_{\mathrm{fy}}} \frac{\sin \left(L_{z}^{j}\left(k_{\mathrm{iz}}-k_{\mathrm{fz}}\right)\right)}{k_{\mathrm{iz}}-k_{\mathrm{f} z}}
$$

where $L_{x}^{j}, L_{y}^{j}$ and $L_{z}^{j}$ are the dimensions of the $j$ th rectangle. In this case the absorption coefficient becomes

$$
\begin{array}{r}
\alpha^{j}(\hbar \omega) \sim \frac{\left|M^{j}\right|^{2}}{\hbar \omega} \int \mathrm{d}^{3} k_{\mathrm{i}} \int \mathrm{d}^{3} k_{\mathrm{f}} h\left(k_{\mathrm{i}}-k_{\mathrm{f}}\right) \delta\left(E_{\mathrm{v}}\left(k_{\mathrm{i}}\right)-E_{\mathrm{c}}^{j}\left(k_{\mathrm{f}}\right)-\hbar \omega\right) \\
\sim \frac{\left|M^{j}\right|^{2}}{\hbar \omega} \int_{E_{\mathrm{z}}-\hbar \omega}^{0} \mathrm{~d} E_{\mathrm{i}} H\left(L_{x}^{j}, L_{y}^{j}, L_{z}^{j}, E_{\mathrm{i}}\right) \rho_{\mathrm{v}}\left(E_{\mathrm{i}}\right) \rho_{\mathrm{c}}^{j}\left(E_{\mathrm{i}}+\hbar \omega\right)
\end{array}
$$

where

$$
H\left(L_{x}^{j}, L_{y}^{j}, L_{z}^{j}, E_{\mathrm{i}}\right)=\int \mathrm{d} \Omega_{k_{\mathrm{i}}} \mathrm{d} \Omega_{k_{\mathrm{f}}} h\left(k_{\mathrm{i}} \hat{k}_{\mathrm{i}}-k_{\mathrm{f}} \hat{k}_{\mathrm{f}}\right)
$$

$\dagger$ The alternative of the smearing due to defect level concentrations is atso less tenable for it requires, as suggested in our study, a specific distribution of defect states-a feature much harder to verify experimentally. 
contains the angular integration over the modulation function, $k_{\mathrm{i}}=\sqrt{-2 m_{\mathrm{v}} E_{\mathrm{i}} / \hbar^{2}}$ and $k_{\mathrm{f}}=\sqrt{-2 m_{\mathrm{c}}\left(E_{\mathrm{f}}+\hbar \omega-E_{\mathrm{g}}^{j}\right) / \hbar^{2}}$. The validity of equations (A3) and (A4) are in fact independent of the precise form of $h$.

Note that if the dimensions of the crystallite are increased, the bulk absorption coefficient is recovered because $h$ limits to a wave-vector-conserving delta function which in turn entails $H$ limiting to an energy delta function that restricts the possible transitions to 'allowed direct'. However, the bulk silicon (i.e. 'allowed indirect') result cannot be recovered because the crystallites are assumed to be direct-gap semiconductors via the zone-folding argument.

If we (temporarily) assume $H\left(L_{x}^{j}, L_{y}^{j}, L_{z}^{j}, E_{\mathrm{i}}\right)$ depends only weakly on the transition energy, the function can be adequately approximated as being independent of $E_{\mathrm{i}}$ and thus taken out of the energy integral. With this approximation the bulk result can no longer be recovered and the absorption coefficient for a single crystallite becomes $\sim \int_{E_{\mathrm{g}}-\hbar \omega}^{0} \mathrm{~d} E_{\mathrm{j}} \rho_{\mathrm{v}}\left(E_{\mathrm{j}}\right) \rho_{\mathrm{c}}^{j}\left(E_{\mathrm{j}}+\hbar \omega\right) \sim(\hbar \omega)^{-1}\left(\hbar \omega-E_{\mathrm{g}}\right)^{2}$. This is in agreement with the more sophisticated theoretical calculations of [10] who observe a square power law near the band edge for photons up to $2.4 \mathrm{eV}$ for a crystallite with diameter $3.86 \mathrm{~nm}$.

Despite its agreement with [10] such an approximation is in fact not correct. If we again consider a rectangular crystallite of side lengths $3.86 \mathrm{~nm}$, the difference between the initial and final momenta (energy) can correspond to a wavelength of up to $\approx 10 \mathrm{~nm}$, indicating that the 'Sinc' function will vary appreciably over the limits of the energy integration in equation (A3). However, by performing this integral numerically we found that the higherorder (non-negligible) corrections to our initial approximation roughly cancel out, leaving a nearly square power law for the absorption coefficient. This seems to be quite insensitive to confinement length and (therefore) crystallite shape. In what follows, we therefore maintain the assumption that $H\left(L_{x}^{j}, L_{y}^{j}, L_{z}^{j}, E_{\mathrm{j}}\right)$ can be taken outside the integral; however, by this we mean that the higher-order corrections roughly cancel.

A slightly different method to our absorption model has been employed by [11, 12] to determine the dielectric function of crystalline nano-sized silicon structures beginning with the bulk silicon Bloch wavefunctions-the 'envelope approximation'. Here, the wavefunction for a given band is a weighted sum of all the Bloch wavefunctions of that band where the modulation function is similar in form to equation (A2). This has the advantage that the bulk silicon absorption coefficient is recovered for large crystallites. We need not consider this sophistication because the typical crystallite sizes that we wish to consider fall well within the quantum regime--having diameters less than $10 \mathrm{~nm}$-where the zone-folding argument is expect to hold.

For optical absorption the incident photons have a kinetic energy ranging between $1.5 \mathrm{eV}$ to $2.0 \mathrm{eV}$. The resolution of such photons is 500 to 600 nanometres and an effective-medium absorption coefficient (the imaginary part of the effective medium dielectric function) can be defined. From basic effective-medium theory it is found that the real and imaginary parts of the effective dielectric function are both a function of the real and imaginary parts of constituent dielectric functions; that is, they are coupled. Fortunately in the optical region for bulk silicon (and therefore for silicon crystallites) the real part is several orders of magnitude larger than the imaginary part entailing that the effective imaginary part of the dielectric function of porous silicon will be strongly proportional to the average of the imaginary part of the crystallite dielectric functions. 
Hence optical absorption experiments measure the mean absorption coefficient of crystallites:

$\alpha_{\mathrm{PS}}(\hbar \omega)=\frac{1}{N} \sum_{j} \alpha^{j} \sim \frac{|M|^{2}}{\hbar \omega} \int_{E_{\mathrm{g}}-\hbar \omega}^{0} \rho_{\mathrm{v}}(E) \frac{1}{N} \sum_{j} H^{j}\left(L_{x}^{j}, L_{x}^{j}, L_{x}^{j}\right) \rho_{\mathrm{c}}^{j}(E+\hbar \omega) \mathrm{d} E$

where the $j$-summation is over all crystallites and $N$ is the number of crystallites that we consider. Here we have included an additional label for the function $H$ since in general this will be strongly dependent on the shape of the crystallite and assume the matrix element near the band edge is independent of the specific characteristics of the crystallite.

Due to the unpredictable local microstructure of porous silicon, the summation (average) over crystallites can be replaced by averages over one or more independent random variables. Since for a given confinement length (band gap) there exists no unique crystallite, the quantities $H^{j}\left(L_{x}^{j}, L_{x}^{j}, L_{x}^{j}\right)$ and $\rho_{\mathrm{c}}^{j}(E)$ will be only weakly correlated. As a first approximation we assume that they are independent by replacing $(1 / N) \sum_{j} H^{j}\left(L_{x}^{j}, L_{x}^{j}, L_{x}^{j}\right) \rho_{c}^{j}(E)$ with $(1 / N) \sum_{j} H^{j}\left(L_{x}^{j}, L_{x}^{j} L_{x}^{j}\right)(1 / N) \sum_{j} \rho_{c}^{j}(E)$, and define

$$
(1 / N) \sum_{j} H^{j}\left(L_{x}^{j}, L_{x}^{j}, L_{x}^{j}\right)=\bar{H}
$$

and (where $\left.\rho_{\mathrm{c}}\left(E, E_{\mathrm{g}}^{j}\right)=\rho_{\mathrm{c}}^{j}(E)\right)$

$$
(1 / N) \sum_{j} \rho_{\mathrm{c}}^{j}(E)=\int_{E_{\mathrm{g}}^{\min }}^{E} \rho_{\mathrm{c}}\left(E, E_{\mathrm{g}}\right) G\left(E_{\mathrm{g}}\right)=\rho_{\mathrm{c}}^{\mathrm{PS}}(E) .
$$

Equation (A7) is thus the average (effective) band-edge conduction density of states for porous silicon. The integration limits in equation (A7) assume $\rho_{\mathrm{c}}\left(E, E_{\mathrm{g}}\right)=0$ for $E \leqslant E_{\mathrm{g}}$, i.e. $\rho^{\mathrm{PS}}(E)$ is constructed from (local) crystallite density of states for which $E_{\mathfrak{g}}<E$ and that there exists a minimum band gap $E_{\mathrm{g}}^{\text {min }}$ larger than that of the bulk which arises from the largest crystallite. Here $G\left(E_{\mathrm{g}}\right)$ is the distribution function for band gaps and is equal to the ratio between the number of crystallites per unit volume per unit band gap and the total number of crystallites per unit volume.

The mean optical absorption coefficient for porous silicon then becomes

$$
\alpha_{\mathrm{PS}} \sim \bar{H} \frac{|M|^{2}}{\hbar \omega} \int \rho_{\mathrm{v}}(E) \rho_{c}^{\mathrm{PS}}(E+\hbar \omega) \mathrm{d} E
$$

\section{References}

[1] Beale M I J, Chew N G, Uren M J, Cullis A G and Benjamin J D 1985 Appl. Phys. Lett. 46 86-8

[2] Cole M W, Harvey J F, Lux R A, Eckart D W and Tsu R 1992 Appl. Phys. Lett. 60 2800-3

[3] Vezin V, Goudeau P, Naudon A, Hamilamaoui A and Bomchil G 1992 Appl. Phys. Lett. 60 2625-7

[4] Schuppler S, Friedman S L, Marcus M A, Adler D L, Xie Y-H, Ross F M, Harris T D, Brown W L, Chabal Y J, Brus L E and Citrin P H 1994 Phys. Rev. Lett. 72 2648-51

[5] Beale M I J, Benjamin J D, Uren M J, Chew N G and Cullis A. G 1985 J. Cryst. Growth 73 622-36

[6] Sagnes I, Halimaoui A, Vincent $G$ and Badoz P A 1993 Appl. Phys. Lett. 82 1155-7

[7] Read A J, Needs R J, Nash K J, Canham L T, Calcott P D J and Qteish A 1992 Phys. Rev. Lett. 69 1232-5

[8] Iyer $S$ and Xie $Y 1993$ Science 260 40-6

[9] Madelung O 1982 Introduction to Solid State Physics 2nd edn (Berlin: Springer) ch 6, pp 252-309

[10] Delerue C, Allan G and Lannoo M 1993 Phys. Rev. B 48 11 024-36

[11] Hybertsen M 1994 Phys. Rev. Lett. 72 1514-7

[12] Xie Y, Hybertsen M S, Wilson W L, Ipri S A, Caver G E, Brown W L, Dons E, Weir B E, Kortan A R, Watson G P and Liddle A J 1994 Phys. Rev. B 49 5386-97

[13] Proot J P, Delerue C and Allan G 1992 Appl. Phys. Lett. 61 1948-50 
[14] Avnir D, Farin D and Pfeifer P 1984 Nature 308 261-3

[15] Unagami T 1980 J. Electrochem. Soc. 127 476-483

- [16] Ohno T, Shiraishi K and Ogawa T 1992 Phys. Rev. Lett. 69 2400-03

[17] Teschke O 1994 Appl. Phys. Lett. 65 1986-8

[18] Takasuka E and Kamei K 1994 Appl. Phys. Lett. 65 484-6

[19] Alexander S and Orbach R 1982 J. Physique Lett. 43 625-31

[20] Englman R 1991 J. Phys.: Condens. Matter 3 1019-53

[21] Stauffer D 1979 Phys. Rep. 54 1-74

[22] Yagil Y, Yosefin M, Bergman D J, Deutscher G and Gadenne 1991 Phys. Rev. B 43 11342-52

[23] Erlebacher J, Sieradzki K and Searson P C 1994 J. Appl. Phys. 76 182-7

[24] Fishman G, Mihalcescu and Romestain R 1993 Phys. Rev. B 48 1464-7 